\title{
Effect of side substituents on thermal stability of the modified chitosan and its nanocomposites with magnetite
}

\author{
Marta Ziegler-Borowska $^{1} \cdot$ Dorota Chełminiak $^{1} \cdot$ Halina Kaczmarek $^{1}$ • \\ Anna Kaczmarek-Kędziera ${ }^{1}$
}

Received: 22 July 2015/ Accepted: 15 January 2016/Published online: 8 February 2016

(c) The Author(s) 2016. This article is published with open access at Springerlink.com

\begin{abstract}
Three different novel chitosan derivatives for medical or biotechnological applications have been successfully obtained by chemical modification of reactive amino and hydroxyl groups in chitosan chain. The modification has led to incorporation of different amount (one to three) of long-distanced amino and imine groups into each repeating unit. These highly functionalized chitosan derivatives were used as a matrix for magnetite nanoparticles. The thermal stability of all obtained chitosan materials has been determined using thermogravimetric analysis in oxidative and inert atmosphere. Chitosan containing two side substituents behaves differently from the other two, which is caused by the significant water uptake. Magnetite causes decrease in thermal stability of studied chitosan derivatives. The highest stability is observed for the nanocomposite obtained from chitosan with three side groups. The changes in the structure of the magnetite core have been observed above $600{ }^{\circ} \mathrm{C}$ in nitrogen. Due to the different competitive reactions occurring in the modified chitosan, the proposed mechanism of thermal degradation is very complex.
\end{abstract}

Keywords Modified chitosan - Side group effect . Thermogravimetric analysis - Effect of water residue . Thermal degradation mechanism

Halina Kaczmarek

halina@chem.umk.pl

1 Faculty of Chemistry, Nicolaus Copernicus University in Toruń, Gagarina 7, 87-100 Toruń, Poland

\section{Introduction}

Modified polysaccharides-valuable biomaterials from renewable resources-play an important role among the polymers for biomedical and pharmaceutical applications. For example, chitosan and its derivatives are the materials intensively studied because of its valuable physicochemical properties and ease of functionalization, which allows to obtain reactive surfaces [1]. Functionalization, i.e., incorporation of the desired type and quantity of functional groups can be achieved by the direct derivatization of macromolecules (what is possible in the presence of the reactive moieties) or by grafting of the monomer or active compound on the polymer surface.

Modern studies of chitosan modification affect many aspects of desirable properties for specific uses. Properly modified biopolymers may exhibit features required for biomedical purposes, for instance demanded ability to adsorption of live cells, proteins, enzymes or drugs. The previous attempts include obtaining chitosan solubility in water, which can be achieved by the simple acetylation process. As reported by Sashiwa et al. [2], the moderate molecular weight and moderate substitution of $\mathrm{N}, \mathrm{O}$-acetyl groups are important factors in obtaining water-soluble chitosan derivative.

Some studies are focused on receiving cross-linked systems, which can find application in the process of removing of toxic impurities from water. Jeon and Höll showed that aminated chitosan beads prepared through reaction with ethylenediamine and carbodiimide have particularly high uptake capacity of mercury ions [3].

The complexation of chitosan by the anionic polysaccharides-heparin and dextran sulfate-allows for the preparation of membranes for hemodialysis characterized by improved blood compatibility [4]. This complexation 
was accompanied by the interpenetration of polyelectrolyte into chitosan matrix swollen in acidic solution and the formation of the semipermeable membrane after alkalization. The content of the modifying agent at the membrane surface can be controlled for proper permeation of biological metabolites.

Sashiwa et al. [5] describe the preparation of the chitosan copolymer graft by acrylic acid via Michael reaction in order to induce the amphiphilic properties. The advantage of chitosan reaction with acrylic acid is that no other solvents than water need to be used.

The reaction of chitosan with selected acid chlorides or acid anhydrides causes the changes in the surface polarity, as confirmed by means of contact angle measurements [6]. The material with the hydrophobic properties has been obtained by the introduction of stearoyl groups into chitosan films, which leads to the promotion of the protein adsorption. The hydrophilic chitosan surface resulting from the reaction with anhydrides was characterized by reduced adsorption of BSA but increased adsorption of lysozyme.

Furthermore, chitosan phosphorylation allows to achieve valuable material for orthopedics due to improving the osteoconduction of polymer-based implants [7]. Phosphate functionalities add cation-exchange properties to chitosan. Moreover, these groups chelate calcium ions inducing the deposition of an apatite-like layer. The presence of both negatively (phosphate) and positively (amine) charged groups provides the amphoteric character of the chitosan.

One recent work describes the effect of electrospinning processing and cross-linking on the thermal degradation of chitosan [8]. For this purpose, the chitosan was dissolved in a mixture of dichloromethane and trifluoroacetic acid solution. Electrospun chitosan has been cross-linked by glutaraldehyde. Resulting material contained ionic groups (even after ethanol treatment) decreasing its thermal stability, which was next slightly improved by cross-linking.

In other publication, the new poly(azo) amino-chitosan compounds were obtained and characterized by thermogravimetry (TG/DTG), differential scanning calorimetry and TG/DTG coupled with FTIR spectroscopy [9]. Although the thermal stability of this new chitosan derivative was lower comparing to the starting chitin-chitosan and $N$-benzyl chitosan, the practical application of such compounds as potential thermal sensors has been proposed.

In addition to the chemical modification of chitosan, the changes in its physicochemical properties can be easily achieved by blending with other synthetic or natural polymers in a physical manner [10-12]. For example, the blends of chitosan with poly(vinyl alcohol), starch or hydroxypropylcellulose with improved thermal stability have been received by this simple method. These new valuable materials may have potential applications in controlled drugs release and tissue engineering.

Recently, new attempts were made to prepare and characterize functionalized chitosan designed to bioligand immobilization. The modified chitosan was demonstrated to be a good matrix for magnetite nanoparticles, which allows to obtain a material with magnetic and biological properties simultaneously, requested in biomedicine and bioseparation techniques [13-17]. It was found that chitosan mixed with poly(quaternary ammonium) salt covering magnetite nanoparticles (NPs) improves the dispersion and increases the activity of immobilized enzymes. As it turned out, it is very useful for the kinetic resolution of $\mathrm{R}$ and $\mathrm{S}$ enantiomers of ibuprofen. Besides, high activity recovery of this nanocomposite material has been found. Valuable chitosan substrate was achieved by enriching the amino groups of the polysaccharide. This modification helped to increase the efficiency of immobilization of lipase and serum albumin as a model protein.

The aim of our work is to investigate the influence of the side substituents containing the amino groups on the thermal stability of such modified chitosan and its nanocomposites containing magnetite $\left(\mathrm{Fe}_{3} \mathrm{O}_{4}\right)$ nanoparticles. For this purpose, three novel chitosan derivatives have been obtained by introducing additional amino groups to the polysaccharide chain in a simple one- or two-step reaction. These derivatives have been employed as the matrix for $\mathrm{Fe}_{3} \mathrm{O}_{4}$. All specimens have been subjected to the thermogravimetric analysis which is a valuable tool of characterization of thermal stability of polymers.

New safe materials based on polysaccharides for biomedical and biotechnological applications are urgently sought. Novel chitosan derivatives proposed in this paper may find practical use, since they fulfill the requirements of this type of materials. The usefulness of these nanocomposites for the immobilization of bioligands has been already confirmed by our preliminary studies, but their thermal stability has been not investigated so far.

\section{Experimental}

\section{Materials}

Chemical reagents (supplied by Sigma) used in this study include: chitosan (CS, low molecular mass, deacetylation degree $75 \%$ ), glutaraldehyde, acetic acid, sodium hydroxide, sodium periodate, ethylenediamine, epichlorohydrin, iron(II) chloride tetrahydrate, iron(III) chloride heksahydrate. All chemicals were analytical grade and used without further purification. 


\section{Chitosan modification}

To obtain one long amine substituent in chain, the chitosan was reacted with glutaraldehyde aqueous solution of ethylenediamine. Materials containing two and three amine substituents were prepared in the reaction of chitosan with epichlorohydrin in alkali solution to form carbonyl groups which were treated with glutaraldehyde and finally with ethylenediamine (Scheme 1). Detailed information on the synthesis of modified chitosan is given below:

Chitosan with one long-distanced free amino group CS-1

Chitosan $0.2 \mathrm{~g}$ was added into $10 \mathrm{~mL}$ of bicarbonate buffer (pH-10). Then, $10 \mathrm{~mL}$ of $5 \%$ glutaraldehyde solution was added and the mixture was mechanically stirred at room temperature for $1 \mathrm{~h}$. The $20 \mathrm{~mL}$ of aqueous solution of ethylenediamine $(2.4 \mathrm{~g}, 40 \mathrm{mmol})$ was added, and the mixture was stirred at room temperature for $2 \mathrm{~h}$. The resulting material was separated from the suspension by filtration, washed five times with deionized water and dried under vacuum at $50{ }^{\circ} \mathrm{C}$ for $24 \mathrm{~h}$.
Chitosan with two long-distanced free amino groups CS-2

Chitosan $0.2 \mathrm{~g}$ was added into $30 \%$ solution of sodium hydroxide $(20 \mathrm{~mL})$ and mechanically stirred at room temperature for $20 \mathrm{~min}$. Epichlorohydrin $(0.2 \mathrm{~mL}, 2.5 \mathrm{mmol})$ was added, and the mixture was stirred at $50^{\circ}$ for $2 \mathrm{~h}$. After cooling to the room temperature, sodium periodate solution ( $0.16 \mathrm{~g}$ in $2.5 \mathrm{~mL}$ of water) was added and the mixture was stirred for $30 \mathrm{~min}$. The $20 \mathrm{~mL}$ of aqueous solution of ethylenediamine $(2.4 \mathrm{~g}, 40 \mathrm{mmol})$ was added, and the mixture was stirred at room temperature for $2 \mathrm{~h}$. The resulting material was separated from the suspension by filtration, washed five times with deionized water and dried under vacuum at $50{ }^{\circ} \mathrm{C}$ for $24 \mathrm{~h}$.

Chitosan with three long-distanced free amino groups CS-3

Chitosan $0.2 \mathrm{~g}$ was added into $30 \%$ solution of sodium hydroxide $(20 \mathrm{~mL})$ and mechanically stirred at room temperature for $20 \mathrm{~min}$. Epichlorohydrin $(0.2 \mathrm{~mL}, 2.5 \mathrm{mmol})$ was added, and the mixture was stirred at $50^{\circ}$ for $2 \mathrm{~h}$. After cooling to the room temperature, sodium periodate solution ( $0.16 \mathrm{~g}$ in $2.5 \mathrm{~mL}$ of water) was added and the mixture was
Scheme 1 Synthesis route of studied chitosan derivatives (on the right-abbreviations used in this work)

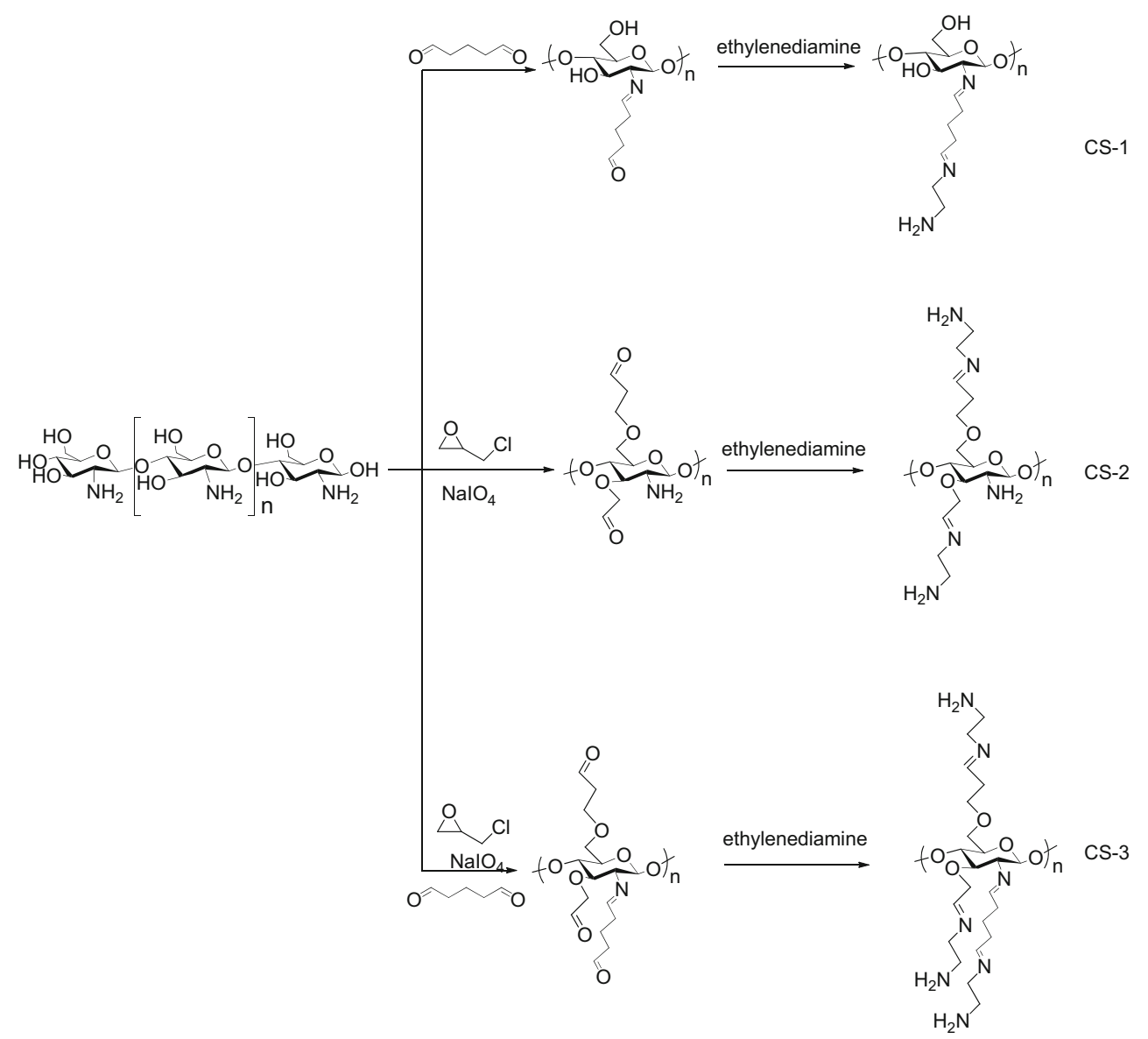


stirred for $30 \mathrm{~min}$. The precipitate was separated by filtration and washed by deionized water for five times. Then, $10 \mathrm{~mL}$ of bicarbonate buffer $\mathrm{pH}-10$ and $10 \mathrm{~mL} 5 \%$ glutaraldehyde solution were added and the mixture was mechanically stirred at room temperature for $1 \mathrm{~h}$. The $20 \mathrm{~mL}$ of aqueous solution of ethylenediamine $(2.4 \mathrm{~g}$, $40 \mathrm{mmol}$ ) was added, and the mixture was stirred at room temperature for $2 \mathrm{~h}$. The resulting material was separated from the suspension by filtration, washed five times with deionized water and dried under vacuum at $50{ }^{\circ} \mathrm{C}$ for $24 \mathrm{~h}$.

The chemical structure of virgin chitosan and its derivatives has been studied by ${ }^{13} \mathrm{C}$ NMR (solid state) spectroscopy using Bruker $700 \mathrm{MHz}$ spectrometer.

\section{Preparation of chitosan derivatives: magnetite nanocomposites}

The magnetic nanoparticles were synthesized by a standard coprecipitation reaction of $\mathrm{Fe}_{3} \mathrm{O}_{4}$ magnetic core with unmodified chitosan as was reported earlier [13]. The chitosan coated on nanoparticles surface was modified in the functionalization reaction by the same way as in the case of pure chitosan without the nanoparticles.

The resulting magnetic material was separated from the suspension by applying a magnet, washed five times with the deionized water and dried under vacuum at $50{ }^{\circ} \mathrm{C}$ for 24-72 h.

The amount of free amino groups on the nanoparticles surface has been estimated by the ninhydrin method which is a standard analysis used for their quantification [18].

\section{$T G$ analysis}

Thermogravimetric analysis was carried out using TA Instrument SDT 2920 Simultaneous DSC-TGA. Measurements were conducted in nitrogen and air atmosphere in the temperature range from room temperature (RT) to $1000{ }^{\circ} \mathrm{C}$. The heating rate and the gas flow rate were $10{ }^{\circ} \mathrm{C} \min ^{-1}$ and $100 \mathrm{~mL} \min ^{-1}$, respectively. The mass of the samples was about $5 \mathrm{mg}$. The figures present curves limited to $800{ }^{\circ} \mathrm{C}$ since in the range of $800-1000{ }^{\circ} \mathrm{C}$ on thermogravimetric curves further changes were not observed. In order to check the effect of moisture on the thermal degradation, the selected samples have been dried at $100{ }^{\circ} \mathrm{C}$ for $1 \mathrm{~h}$ immediately before measurement.

\section{Results}

\section{General characterization}

Chemical modification of chitosan (CS) allowed for the introduction of 1-3 (assigned as CS-1, CS-2, CS-3) additional amino groups away from the polysaccharide backbone. The modification was a two-step reaction, which is shown in Scheme 1.

Magnetite nanocomposites, after precipitation from acidic solutions by base, become fine powders with a white or yellowish color, insoluble in water.

The ${ }^{13} \mathrm{C}$-NMR spectra confirming the chemical structure of obtained chitosan derivatives are presented in Fig. 1. The main signals $(\delta$ in ppm) in pure chitosan (Fig. 1a) appear at 58 (C2, C6); 75 (C3, C5); 84 (C4); 105 (C1). This spectrum shows a small signal at $22 \mathrm{ppm}$ due to incomplete deacetylation (deacetylation degree of studied chitosan was $75 \%)$.

${ }^{13} \mathrm{C}$ NMR analysis of chitosan derivatives (CS-1, CS-2, CS-3) revealed that spectra exhibited new clear peak at $170-180 \mathrm{ppm}$, attributed to $\mathrm{C}=\mathrm{N}$ bond in polysaccharide side chain, which is absent in virgin, unmodified chitosan (Fig. 1b-d). In CS-3 spectrum, the differences between C2 and C6 carbons from chitosan chain are stronger. Two separated signals from these carbons (at 57 and $61 \mathrm{ppm}$ ) are observed as a result of different chemical surrounding. Assignment of NMR bands to proper groups is based on the work of Heux et al. [19].

The lack of solubility of the obtained chitosan derivatives rules out the use of other analytical techniques such as NMR in solution or GPC.

An additional chemical analysis (ninhydrin method [18]) has been done to confirm the structure of obtained nanocomposites of chitosan derivatives with magnetite. This method allowed to determine the content of primary amine groups on nanoparticle surface, which varies between $3.15 \quad\left(\mathrm{Fe}_{3} \mathrm{O}_{4}\right.$-CS-1), $5.3 \quad\left(\mathrm{Fe}_{3} \mathrm{O}_{4}\right.$-CS-2) and $8.34 \mathrm{mM} \mathrm{g}^{-1}\left(\mathrm{Fe}_{3} \mathrm{O}_{4}-\mathrm{CS}-3\right)$. The high amount of $\mathrm{NH}_{2}$ groups was observed on $\mathrm{Fe}_{3} \mathrm{O}_{4}-\mathrm{CS}-3$ surface as it was expected.

In the previous microscope research (by SEM) of magnetite precipitated by this method, the size of the nanoparticles formed was estimated at several tens of nm, which allows the use of the term "nanocomposite" in relation to our systems. It should be pointed out that CS-1 and CS-2 chitosan derivatives are new compounds, undescribed in the literature, while CS-3 has been investigated in our group as a component of a mixture with poly(quaternary ammonium) salt [14].

The magnetic properties of the obtained systems may be easily observed by using an ordinary magnet applied to the outside of the vessel containing the dispersion of nanoparticles.

The presence of additional amine groups in each repeating units of chitosan is very important from point of view of application in biomedicine. These groups are responsible for interaction with functional groups of enzymes, peptides or drugs. Moreover, in modified 
Fig. $1{ }^{13} \mathrm{C}$ NMR spectra of a pure chitosan, b CS-1, $\mathbf{c}$ CS-2 and d CS-3
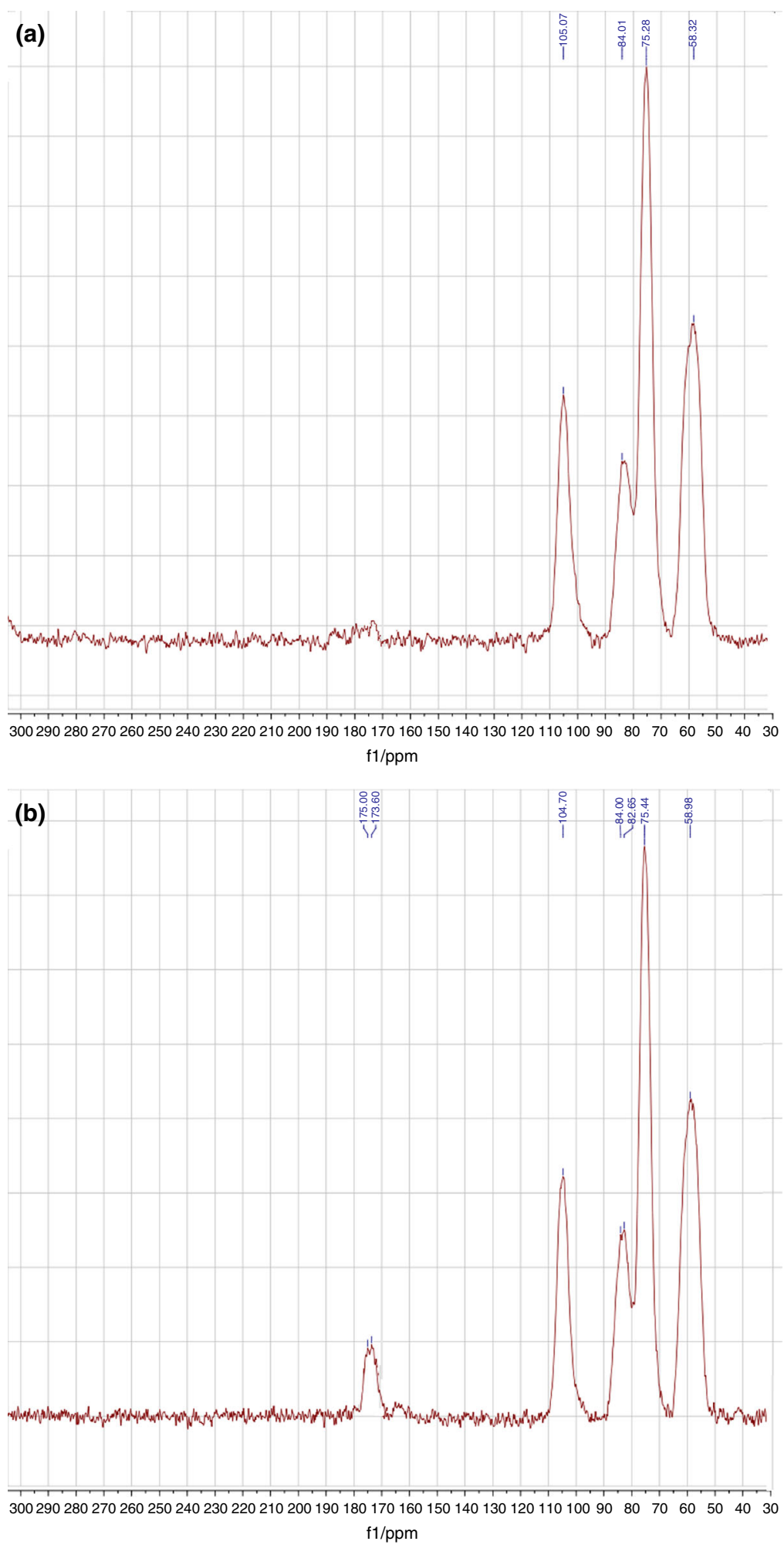

chitosans these side groups are "pending" and distanced from the backbone which leads to their higher mobility contrary to $\mathrm{NH}_{2}$ directly joined to glycoside ring. Such mobility leads to easy penetration in the phase of interacting material and formation of stable bonds necessary for immobilization. 
Fig. 1 continued

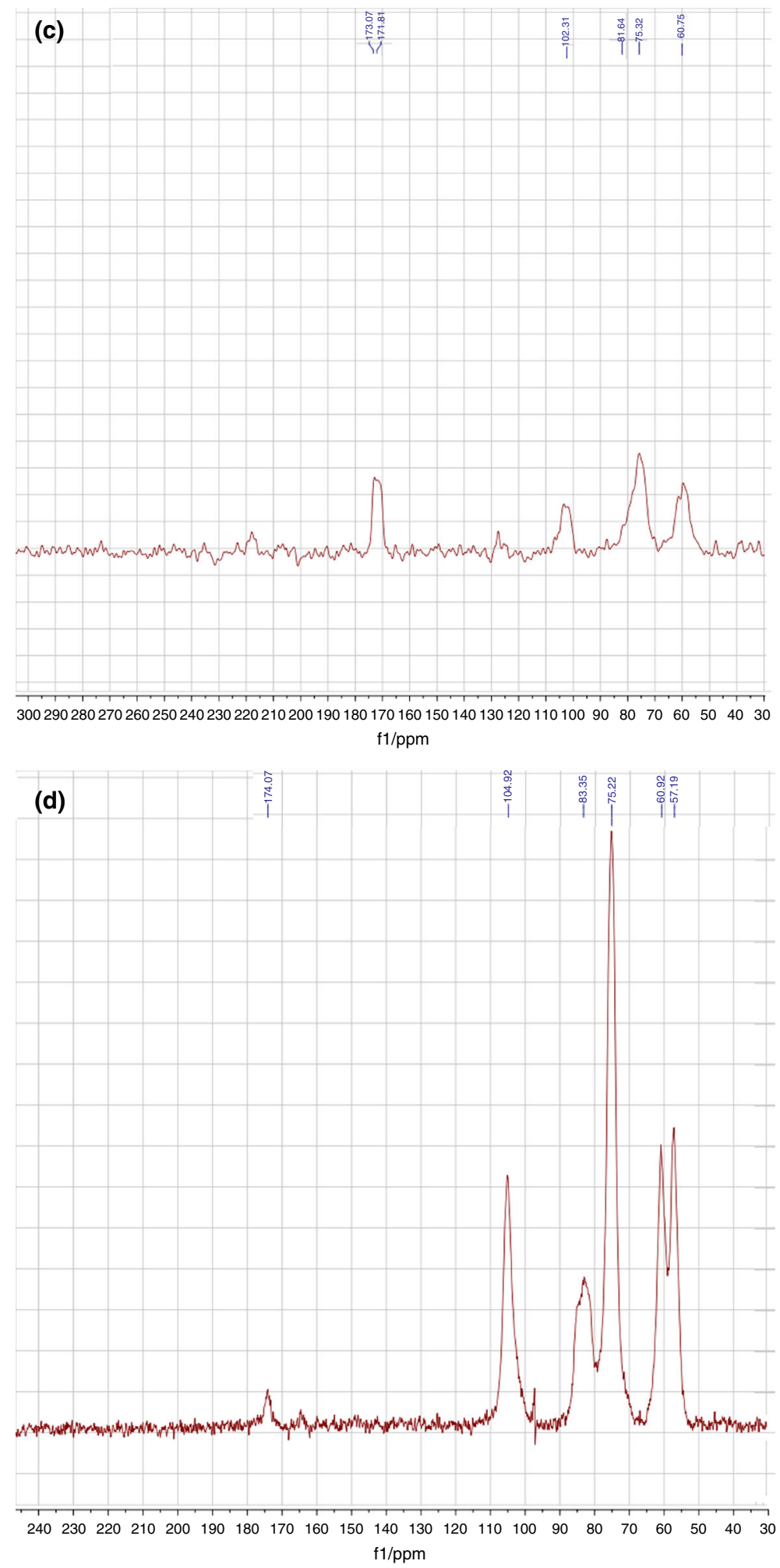

Thermogravimetric analysis in nitrogen

The overall thermogravimetric decomposition process of modified chitosan derivatives and their nanocomposites with $\mathrm{Fe}_{3} \mathrm{O}_{4}$ is presented in Fig. 2a, b. Additionally, Fig. 2a contains thermogravimetric curve of origin (unmodified) chitosan for comparison.

It is generally accepted that the reference CS undergoes the thermal decomposition in two stages, which are well explained in the literature $[10,12,17,20]$. The first stage, 

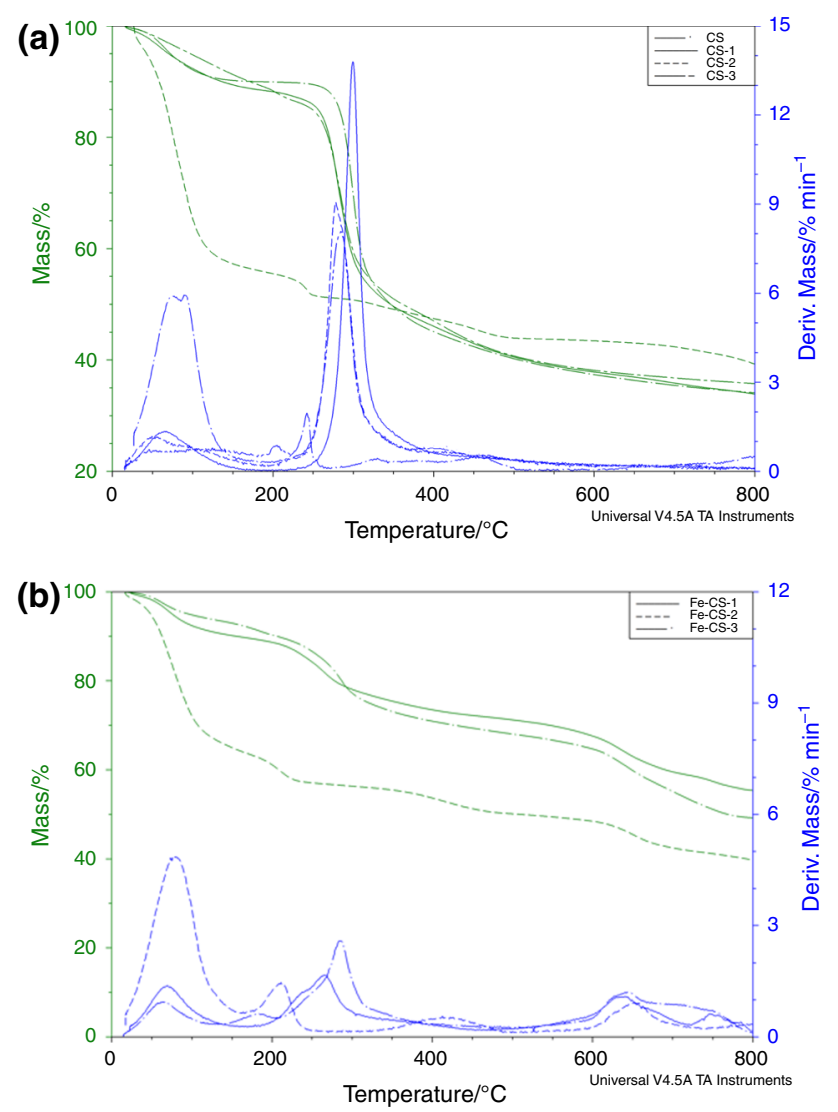

Fig. 2 Thermogravimetric curves (TG and DTG) of virgin and modified chitosan (CS, CS-1, CS-2, CS-3) (a) and their nanocomposites with magnetite $(\mathbf{b})$ in nitrogen atmosphere

occurring at lower temperatures $\left(20-150{ }^{\circ} \mathrm{C}\right)$, is assigned to releasing of adsorbed water, which is obvious because of the CS hydrophilicity. The intensive destruction of CS macromolecules takes place in the second (main) stage in the temperature range of $200-500{ }^{\circ} \mathrm{C}$. It was repeatedly confirmed that the main chain scission, abstraction of side groups and ring opening reactions occur in this step. The carbonaceous residue in $\mathrm{N}_{2}$ atmosphere is $\sim 34 \%$ at $800{ }^{\circ} \mathrm{C}$ (but even at $1000{ }^{\circ} \mathrm{C}$ the decay is not completed).

Thermogravimetric curves of modified CS-1 and CS-3 specimens also exhibit two clear steps in the studied temperature range. First step starts at room temperature and proceeds to about $150{ }^{\circ} \mathrm{C}$, and the second extends to around $400{ }^{\circ} \mathrm{C}$. Finally, at higher temperatures $\left(>400{ }^{\circ} \mathrm{C}\right)$ the decomposition is continued and systematic mass loss without any maximum rate is observed. CS-2 shows the exceptional thermal degradation course. At least three steps (moreover, each of them is complex) can be found on the TG curve and the DTG.

Thermogravimetric profiles of nanocomposites vary from the chitosan one. TG curves of nanocomposites with $\mathrm{Fe}_{3} \mathrm{O}_{4}$ (Fig. 2b) show three-four steps of thermal degradation. The meaningful difference is caused by high residue at the end of experiment due to the inorganic part presence.

In order to interpret the differences in the behavior of the studied samples during heating, major thermal parameters were determined from the TG curve. These data, including the corresponding values for the reference CS, are summarized in Table 1.

The first step for all curves, associated with the release of adsorbed water, does not relate to a chemical change in the structure of the polymer during heating. However, the determined $\mathrm{H}_{2} \mathrm{O}$ content can indicate a various susceptibility of chitosan specimens to water sorption. It should be pointed out that all samples were thoroughly dried under identical conditions (to a constant mass) but the mass losses observed in the first step are different for the studied chitosan derivatives. As can be seen from Table 1, the highest hydrophilicity is shown by the CS-2 sample (44\% mass loss corresponding to the removal of water). Also in the case of nanocomposites, the $\mathrm{Fe}_{3} \mathrm{O}_{4}$-CS-2 was the most hydrophilic (35\%). In other samples, the water content is in the range of $7-12 \%$, which is typical for polysaccharides.

The following discussion focuses on the changes during the second, main stage of degradation above $140{ }^{\circ} \mathrm{C}$. On the basis of $T_{\mathrm{o}}$ and $T_{\max }$, it can be seen that the thermal stability of modified chitosan relative to the unmodified one decreases but no simple correlation between the amount of side groups and these parameters can be found. Nanocomposites also exhibit lower values of $T_{\mathrm{o}}$ and $T_{\max }$ than pure chitosan.

Comparing nanocomposites with the respective chitosan derivatives, a slight decrease in $T_{\mathrm{o}}$ in all cases in the presence of $\mathrm{Fe}_{3} \mathrm{O}_{4}$ has been observed. Interestingly, the chitosan containing the three groups (CS-3) has the same $T_{\max }$ in the presence of magnetite.

At the same time, it can be seen that the mass loss $(\Delta m)$ at this second stage is greater for samples that show less $\Delta m$ in the first step. However, such a relationship has not been found for chitosans with magnetite. The last step of nanocomposites thermal decomposition, occurring above $600{ }^{\circ} \mathrm{C}$, can be assigned to changes in the inorganic core. According to the literature, magnetite at this temperature is converted to $\gamma$-hematite (maghemite, $\mathrm{Fe}_{2} \mathrm{O}_{3}$ ) or nonstechiometric wustite $\mathrm{Fe}_{1-x} \mathrm{O}$ [21-25]. Although this reaction seems more probable in the presence of air (it requires the presence of an oxidant), one may also assume that this transformation can involve oxygen atoms from CS chains or oxidizing degradation products.

Although it is expected that the modified chitosan will be applied at moderate temperatures, for full characterization of new materials, the thermal analysis in broad range of temperature is needed. Such knowledge is particularly important in the case of sterilization of product in autoclaves or recycling, i.e., in the recovery of magnetite 
Table 1 Thermal parameters of the obtained magnetite nanoparticles from thermogravimetric analysis in nitrogen atmosphere

\begin{tabular}{|c|c|c|c|c|c|c|c|c|c|}
\hline \multirow[t]{2}{*}{ Sample } & \multicolumn{2}{|c|}{ First stage } & \multicolumn{3}{|c|}{ Second stage } & \multicolumn{3}{|c|}{ Third stage } & \multirow[t]{2}{*}{ Residue at $800{ }^{\circ} \mathrm{C} / \%$} \\
\hline & $T_{\max } /{ }^{\circ} \mathrm{C}$ & $\Delta m / \%$ & $T_{\mathrm{o}} /{ }^{\circ} \mathrm{C}$ & $T_{\max } /{ }^{\circ} \mathrm{C}$ & $\Delta m / \%$ & $T_{\mathrm{o}} /{ }^{\circ} \mathrm{C}$ & $T_{\max } /{ }^{\circ} \mathrm{C}$ & $\Delta m / \%$ & \\
\hline $\mathrm{CS}$ & 64 & 10 & 210 & 298 & 44 & - & $\mathrm{a}$ & 12 & 34 \\
\hline CS-1 & 52 & 11 & 186 & 277 & 43 & 400 & 461 & 12 & 34 \\
\hline $\mathrm{CS}-2$ & 85 & 44 & 187 & 242 & 5 & 285 & 389 & 12 & 39 \\
\hline $\mathrm{CS}-3$ & a & 12 & 190 & $\begin{array}{l}284 \\
421\end{array}$ & 40 & - & a & 12 & 36 \\
\hline $\mathrm{Fe}_{3} \mathrm{O}_{4}-\mathrm{CS}-1$ & 72 & 10 & 163 & 263 & 17 & 459 & 637,763 & 18 & 55 \\
\hline $\mathrm{Fe}_{3} \mathrm{O}_{4}-\mathrm{CS}-2$ & 79 & 35 & 160 & 212,417 & 15 & 528 & 652 & 10 & 40 \\
\hline $\mathrm{Fe}_{3} \mathrm{O}_{4}-\mathrm{CS}-3$ & 62 & 7 & 140 & $\begin{array}{l}284 \\
181\end{array}$ & 28 & 550 & 644 & 16 & 49 \\
\hline
\end{tabular}

$C S$ chitosan, $C S$ - 1 chitosan with one long-distanced amino group, $C S$ - 2 chitosan with two long-distanced amino groups, $C S$ - 3 chitosan with longdistanced amino groups

${ }^{a}$ Lack of clear inflection point on TG and no maximum on DTG; mass decreases gradually

nanoparticles by removing the protective coating of chitosan in the process of pyrolytic decomposition.

\section{Thermogravimetric analysis in air}

The degradation of CS reference in air occurs in three stages (Fig. 3a, b; Table 2), which is consistent with our previous published results [17]. The first stage is also associated with the removal of moisture, while the second (main) step includes both decomposition and oxidation reactions of $\mathrm{CS}$. In the last stage, there is almost a complete degradation of intermediates generated earlier at lower temperatures. It should be added that the mass loss is almost total-residue in $800{ }^{\circ} \mathrm{C}$ in this case is only $2 \%$, in contrast to the degradation in an inert atmosphere $\left(\mathrm{N}_{2}\right)$ with the remaining organic fraction of about $34 \%$. This is reasonable because the polysaccharides are burned in the presence of $\mathrm{O}_{2}$ with $\mathrm{CO}_{2}$ and $\mathrm{H}_{2} \mathrm{O}$ secretion.

The obtained CS-1 and CS-3 derivatives exhibit similar behavior, but a CS-2 sample is an exception (just as it was in nitrogen). It is worth noting here that the volatilization is also almost complete in the case of CS-1, CS-3 and unmodified (reference) CS heated in the air.

The course of degradation in CS-2 deviates significantly from the other samples. Apart from a much higher moisture content released in several stages, which shows the different durability of water binding, the sample exhibits no decomposition stage within $250-350{ }^{\circ} \mathrm{C}$ (as observed for CS, CS-1 and CS-3). In contrast, the approximately $10 \%$ mass loss appears at $650-700{ }^{\circ} \mathrm{C}$ (step with a significant rate), which is not observed in other chitosan derivatives or even in this sample heated in nitrogen. Significant carbon residue in this case at $800{ }^{\circ} \mathrm{C}(52 \%)$ shows the creation of permanent, nonvolatile structures which are not burned at such high temperatures. It can be assumed that they are
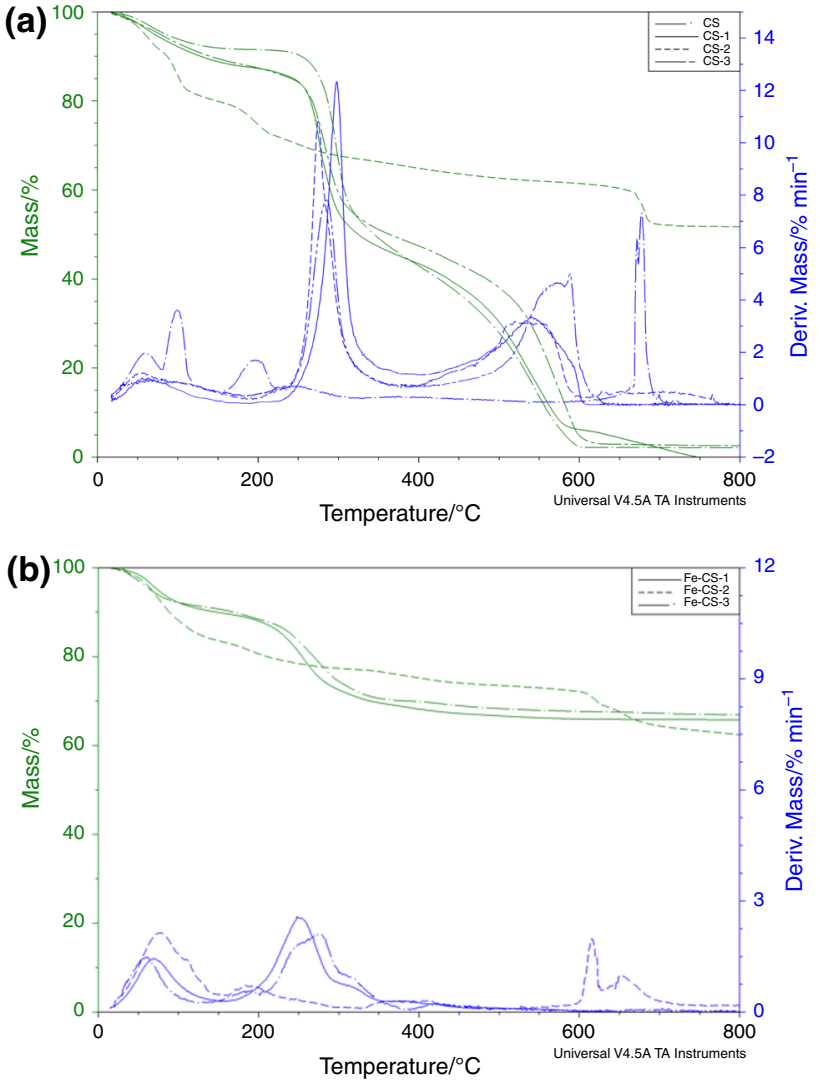

Fig. 3 Thermogravimetric curves (TG and DTG) of virgin and modified chitosan (CS, CS-1, CS-2, CS-3) (a) and their nanocomposites with magnetite (b) in air atmosphere

nitrogen-enriched cross-linked or polycyclic aromatic structures.

Nanocomposites heated in the air confirm the unusual behavior of CS-2. This polymer-coated magnetite also has a high water content $(17 \%)$, slight mass loss in the range of 
Table 2 Thermal parameters of the obtained magnetite nanoparticles from thermogravimetric analysis in air atmosphere

\begin{tabular}{|c|c|c|c|c|c|c|c|c|c|}
\hline \multirow[t]{2}{*}{ Sample } & \multicolumn{2}{|c|}{ First stage } & \multicolumn{3}{|c|}{ Second stage } & \multicolumn{3}{|c|}{ Third stage } & \multirow{2}{*}{$\begin{array}{l}\text { Residue at } \\
800{ }^{\circ} \mathrm{C} / \%\end{array}$} \\
\hline & $T_{\max } / \mathrm{C}$ & $\Delta m / \%$ & $T_{\mathrm{o}} /{ }^{\circ} \mathrm{C}$ & $T_{\max } /{ }^{\circ} \mathrm{C}$ & $\Delta m / \%$ & $T_{\mathrm{o}} /{ }^{\circ} \mathrm{C}$ & $T_{\max } /{ }^{\circ} \mathrm{C}$ & $\Delta m / \%$ & \\
\hline CS & 63 & 9 & 220 & 298 & 48 & 395 & 541 & 41 & 2 \\
\hline CS-1 & 54 & 10 & 186 & 274 & 44 & 399 & 535 & 44 & 2 \\
\hline $\mathrm{CS}-2$ & 99 & 19 & 142 & 196 & 14 & 350 & 676 & 15 & 52 \\
\hline $\mathrm{CS}-3$ & 80 & 12 & 200 & $\begin{array}{l}285 \\
216\end{array}$ & 40 & 400 & 570 & 45 & 3 \\
\hline $\mathrm{Fe}_{3} \mathrm{O}_{4}-\mathrm{CS}-1$ & 68 & 10 & 180 & 248 & 17 & 300 & 314 & 7 & 66 \\
\hline $\mathrm{Fe}_{3} \mathrm{O}_{4}-\mathrm{CS}-2$ & 72 & 17 & 163 & 263 & 10 & 459 & $\begin{array}{l}637 \\
763\end{array}$ & 10 & 63 \\
\hline $\mathrm{Fe}_{3} \mathrm{O}_{4}-\mathrm{CS}-3$ & 62 & 9 & 130 & $\begin{array}{l}275 \\
191\end{array}$ & 21 & 390 & 423 & 3 & 67 \\
\hline
\end{tabular}

200-400 ${ }^{\circ} \mathrm{C}$ and an additional decomposition stage at $600-700{ }^{\circ} \mathrm{C}$ (not present in the other samples). This last step is also complex, which can be clearly seen in the DTG curve. The observed peaks are shifted to lower temperatures comparing to CS-2 alone. This means that the stable, crosslinked residue differs in the structure comparing to product formed in $\mathrm{N}_{2}$ (in the air it may contain more oxygen atoms).

It should be emphasized that in other nanocomposites $\left(\mathrm{Fe}_{3} \mathrm{O}_{4}-\mathrm{CS}-1\right.$ and $\left.\mathrm{Fe}_{3} \mathrm{O}_{4}-\mathrm{CS}-3\right)$ no evidence of any peaks in the DTG curves can be found that could be attributed to the changes occurring in the magnetite. It means that during the thermal removal of the protective polymer layer in air atmosphere, the magnetic particles retain their properties and are suitable for reuse.

\section{Effect of water content}

The CS-2 sample has the highest water content, which proves its high sorption capability and relatively permanent $\mathrm{H}_{2} \mathrm{O}$ bonding by macromolecules in comparison with two other chitosan derivatives: CS-1 and CS-3. This is a rather surprising result. It should be added that in the questionable cases, i.e., in the samples characterized by a high content of moisture $(>10 \%)$, TG analysis was repeated several times. The excellent properties of CS-2 distinguishing it from other derivatives were also proved by biological activity studies. From the point of view of biomedical applications, complete drying of samples is undesirable, but in the case of thermal decomposition, the moisture can have a significant impact on this process. At elevated temperatures, water may cause hydrolysis of bonds leading to the shortening of the side substituents.

The water effect on the course of thermal degradation of modified chitosan in nitrogen atmosphere is presented on the base of thermogravimetric curves of CS-2 recorded for different drying steps (Fig. 4). As can be seen, this specimen dried in vacuum at $50{ }^{\circ} \mathrm{C}$ during 24 and $72 \mathrm{~h}$, despite

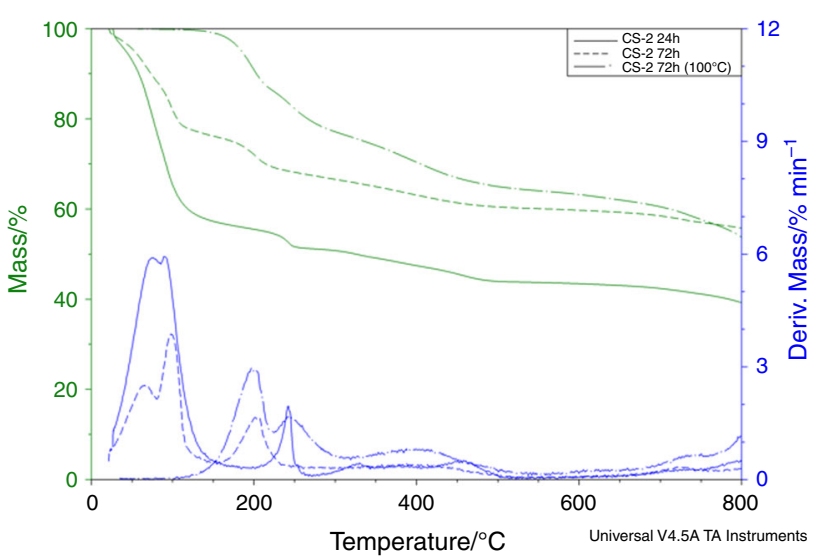

Fig. 4 Thermogravimetric curves of CS-2 in nitrogen atmosphere illustrating effect of water content

constant mass, still contains about 30 and $40 \%$ moisture, respectively. It is difficult to remove the water residue at lower temperatures. Only the increase in the temperature to $100{ }^{\circ} \mathrm{C}$ just before thermogravimetric analysis allows for the precise removal of the $\mathrm{H}_{2} \mathrm{O}$ residual. The presence of water results in a multi-step decomposition of CS-2 in a nitrogen atmosphere (3-4 clear peaks appear in DTG). The sample completely free of water also decomposes in three stages but at higher temperatures $\left(T_{\max }=200,250\right.$, $\left.400{ }^{\circ} \mathrm{C}\right)$. It is interesting that in the sample with the highest water content, the main stage of decomposition occurs at approximately $245^{\circ} \mathrm{C}$. This indicates a change in the thermal degradation mechanism. It can be concluded that the residual water promotes the hydrolytic decomposition at higher temperatures.

\section{Overview of the structure and course of the thermal degradation of modified chitosan derivatives}

The novel chitosan derivatives contain one-three long substituents ended by amine groups. Moreover, in each side 
chain, $\mathrm{C}=\mathrm{N}$ imine moiety is present. This type of functional group can participate in numerous reactions. In the side chain of CS-1, two imine groups are present; in CS-2, which have two side chains, only one $\mathrm{C}=\mathrm{N}$ exists in each substituent, while in CS-3-both types of side groups are substituted. At the same time, the number of amino groups varies from 1 to 3 (in CS-1, CS-2 and CS-3), respectively. The side groups are joined to chitosan macrochain via oxygen atom in position $\mathrm{C} 3$ and $\mathrm{C} 6(\mathrm{CS}-2)$ or via nitrogen atom as in CS-1. The chitosan with highest functionality (CS-3) contains both types of connections. CS-1 still has in its structure the two $\mathrm{OH}$ groups in each glycoside unit.

The amine and hydroxyl groups are responsible for hydrogen bond formation between macromolecules. Typical hydrogen bonds are formed between amine end groups $\left(\mathrm{NH}_{2} \cdots \mathrm{NH}_{2}\right)$ in CS-2 and CS-3, while hydrogen bridges via hydroxyl groups ( $\mathrm{HO} \cdots \mathrm{HO}$ or $\left.\mathrm{OH} \cdots \mathrm{NH}_{2}\right)$ are possible only in the case of CS-1. Also adsorbed water participates in hydrogen bond formation.

The chemistry of imines has been described in several works; however, still no information on their thermal decomposition is available. Imine contains electron-rich nitrogen atoms which acts as a typical nucleophile. In addition, the imine has also an electrophilic nature due to the carbon atom in $\mathrm{C}=\mathrm{N}$ group [26-28]. The chemical properties of imines are often compared to the carbonyl compounds. However, nitrogen is less electronegative (3.0 in Pauling scale) than oxygen (3.5); thus, $\mathrm{N}=\mathrm{C}$ is less polarized than $\mathrm{C}=\mathrm{O}$, and in consequence, imines are less electrophilic than aldehydes and ketones.

The imines are more stable and less reactive than the primary amines; thus, mainly $\mathrm{NH}_{2}$ end groups participate in the reactions occurring in studied systems in the presence of other reagents.

The possible reactions of imines, described in the literature [26-28], are reversible exchange and metathesis. Such reactions can occur in heated samples; hence, the various chitosan derivatives can appear in the degradation products. However, it seems that the water that causes hydrolysis has a greater influence on the thermal degradation (Scheme 2). The imine hydrolysis can lead to the formation of fragments containing new amine and aldehyde groups. As can be seen from Scheme 2, among the low molecular mass degradation products the diamines, dialdehydes and aminoaldehydes can be found. They are active chemical compounds, which may thus cause secondary reactions in partially degraded chitosan.

The above reactions account for the examples of the formation of various degradation products; therefore, it is expected that the resulting mixture is very complex. Moreover, organic products of hydrolysis undergo decomposition in the subsequent stages of degradation.
Another type of imine reaction is cycloaddition with alkenes or dienes, which have been explored by few research groups [26-28]. Although unsaturated and aromatic degradation products are formed in thermally degraded chitosan (mainly in the absence of oxygen), the above-mentioned reaction is unlikely because it requires Lewis acid or special catalyst to activate the imine.

The mechanism of thermal degradation of pure chitosan has been discussed previously in detail [19, 29-31]. As generally accepted, the thermal degradation of chitosan is a free radical mechanism. The homolytic fission of chemical bonds leads to fragments containing elements from which macromolecules were built. In our case, compounds containing nitrogen atoms appear besides the carbon- and oxygen-containing species.

The processes occurring in the samples of modified chitosan can be much more diversified, because the thermal decomposition can also take place in the side chains.

Thus, in addition to the typical reactions of chitosan such as the scission of glycoside bonds, ring opening, side group abstraction accompanying by the elimination of volatile products, the destruction of side chains in different places is expected. Comparing the energies of chemical bonds (in $\mathrm{kJ} / \mathrm{mol}$ ) occurring in the studied samples: $\mathrm{C}-\mathrm{C}$ (345), C-O (350), C-N (290), C=N (615) [32], it can be concluded that in the weakest point (i.e., in carbon-nitrogen moiety), the cracking takes place easily (Scheme 3).

At higher temperatures, the degraded chitosan residue undergoes carbonization and final mixture of products depends on the atmosphere in which the thermal decomposition was carried out [29]. In the air atmosphere, at the process beginning, the oxidation competes with the fragmentation. Finally, the entire combustion dominates, during which mainly water, carbon and nitrogen oxides $(\mathrm{CO}$, $\mathrm{CO}_{2}, \mathrm{NO}_{\mathrm{x}}$ ) are emitted.

Furthermore, free radicals formed during the thermolysis can recombine leading to cross-linking chains; therefore, the volatilization is not full. There are few possibilities of cross-linking in chitosan derivatives, which can include direct link of partially decomposed CS particles. For instance, the recombination of any macroradicals (some of them are shown in Scheme 2) leads to the formation of bonds joining macrochains. It can be assumed that covalent cross-links are also formed when side chains react due to the unsaturated $\mathrm{C}=\mathrm{N}$ bonds presence (Scheme 4).

Such an addition reaction can occur between the groups from the same (intramolecular process) or different (intermolecular process) molecules. Although intermolecular cross-linking usually reinforces the polymer structure, the formation of four-membered rings seems not very stable at elevated temperature due to the tension of this cyclic structure. 


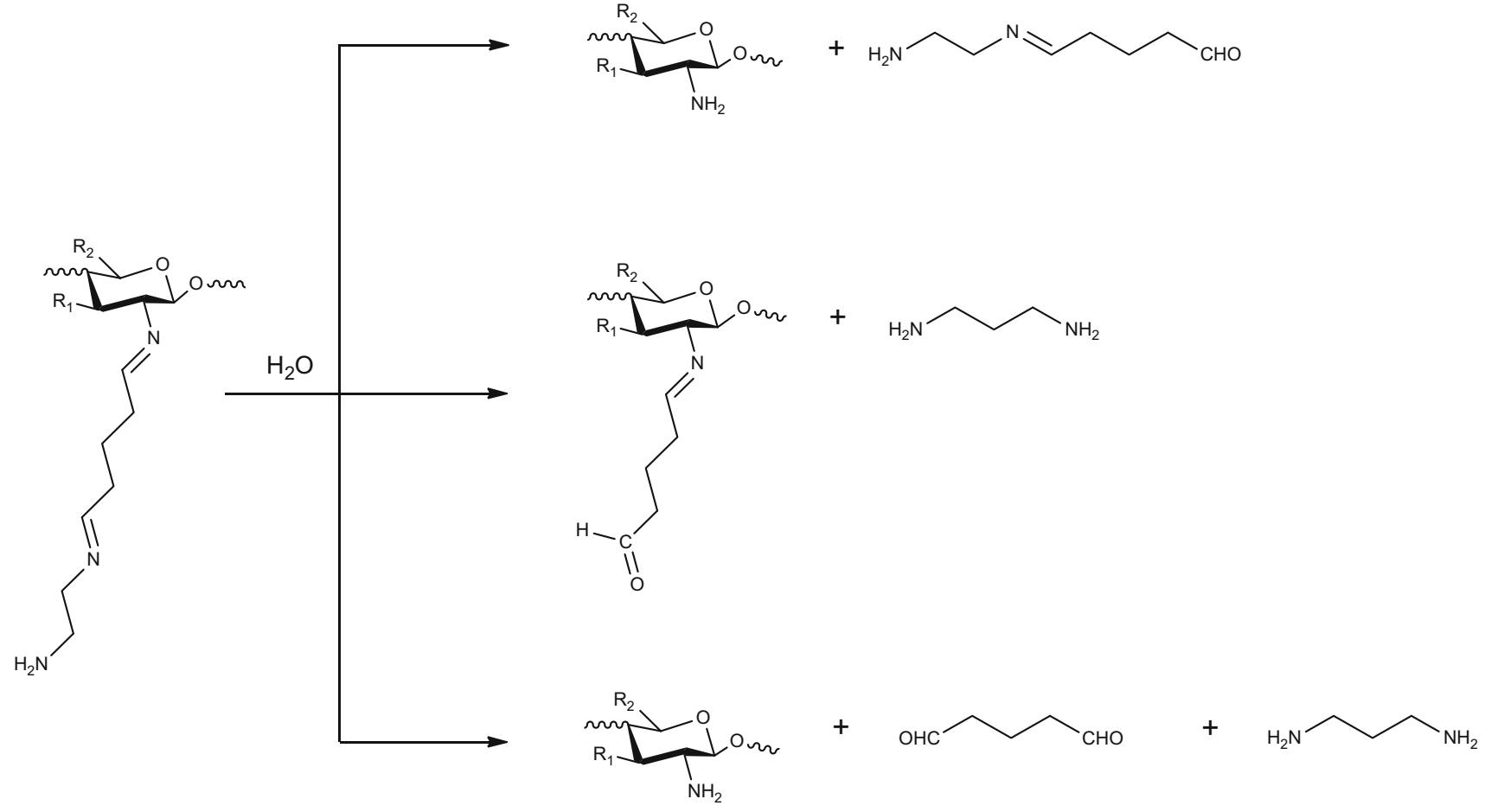

Scheme 2 Hydrolysis of modified chitosan $\left(R_{1}\right.$ and $R_{2}$ substituents shown in Fig. 1)

Differences observed in studied CS-1, CS-2 and CS-3 samples indicate that the thermal stability is dependent on the type and number of functional groups in side substituents.

Lower thermal stability of chitosan derivatives comparing to the raw unmodified chitosan suggests that the formed free radicals can attack the polysaccharide chains. The small, mobile fragments, e.g., $\cdot \mathrm{NH}_{2}, \cdot \mathrm{CH}_{2}-\mathrm{CH}_{2}-\mathrm{NH}_{2}$ (see Scheme 3), are particularly active.

Chitosan nanocomposites exhibit further slight decrease in thermal stability comparing to CS alone, which suggests the catalytic action of $\mathrm{Fe}_{3} \mathrm{O}_{4}$.

Finally, one should explain the different behavior of CS2 during heating, compared to the other modified CS samples. It can be concluded that fragmentation of main and side chains is a dominant process in CS- 1 and CS-3. These reactions also occur in CS-2, but there is a reduction in stability due to hydrolysis of imino groups in the side chains. Surprisingly, however, in this sample heated in an air atmosphere stable structures were formed whose degradation occurred only above $600{ }^{\circ} \mathrm{C}$. This indicates the formation of the highly cross-linked, compact, dense carbonaceous residue or even the occurrence of the graphitization-like process.

The latter hypothesis was confirmed by comparing the CS-2 and graphite TG curves, registered in the air. A clear peak in the range of $650-700{ }^{\circ} \mathrm{C}$ may correspond to a combustion process of graphite [33] or graphite-like structures. In the presence of magnetite, the decomposition of this carbonaceous structure is slightly earlier $\left(600-650{ }^{\circ} \mathrm{C}\right)$, which proves the catalytic impact of iron oxide. It should be noted that in other samples, characterized by a lower water content, such stable structure does not arise. The evidence of the creation of unsaturated and aromatic structures in pyrolyzed CS has been provided by FTIR spectroscopy studies [29].

The dominant water sorption and privileged "graphitization" in the CS-2 sample can be explained by its chemical structure. Probably the two relatively large side groups constitute the system, which allows water molecules to penetrate and to form the relatively stable hydrogen bonds. On the other hand, too small proportion of the side groups in the CS-1 also does not favor the growth of such permanent water binding. Moreover, in the CS-3 the too large steric hindrance appears.

Summarizing, during the thermal degradation of novel, functionalized derivatives of chitosan and their magnetic nanocomposites, the following reactions should be considered:

(a) In inert atmosphere $\left(\mathrm{N}_{2}\right)$ - scission of main chain accompanying by the destruction of side groups, cross-linking and formation of carbonaceous residue resistant to the decomposition at high temperature (mainly endothermic processes);

(b) In air atmosphere-dominant oxidation reaction leading to the formation of volatile products 


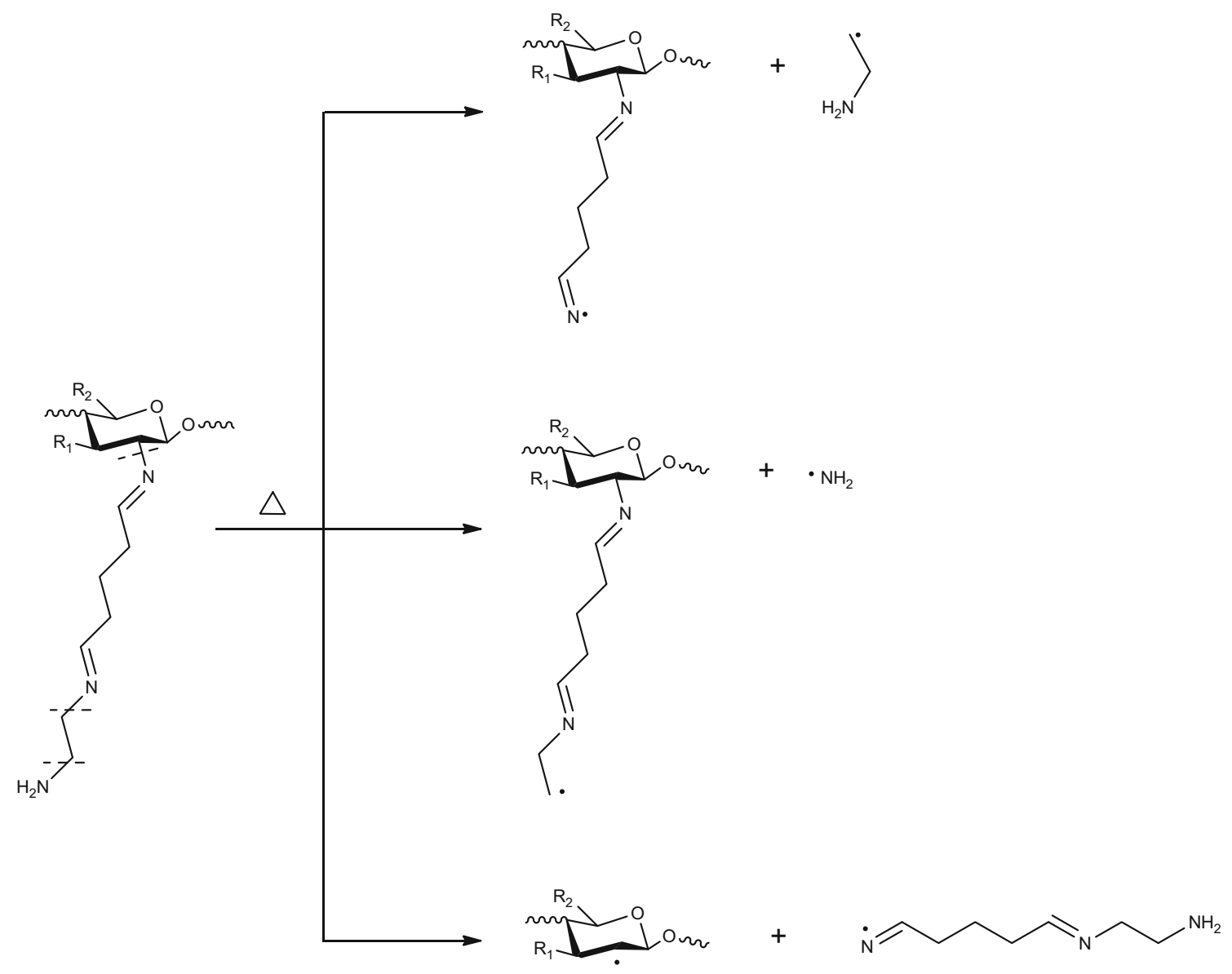

Scheme 3 Thermal decomposition of chitosan derivatives $\left(\mathrm{R}_{1}\right.$ and $\mathrm{R}_{2}$ substituents shown in Fig. 1, dashed lines show the weak points susceptible to breaking)

Scheme 4 Example of crosslinking via addition reaction of imine groups

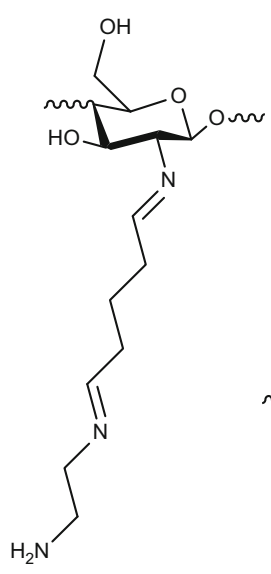

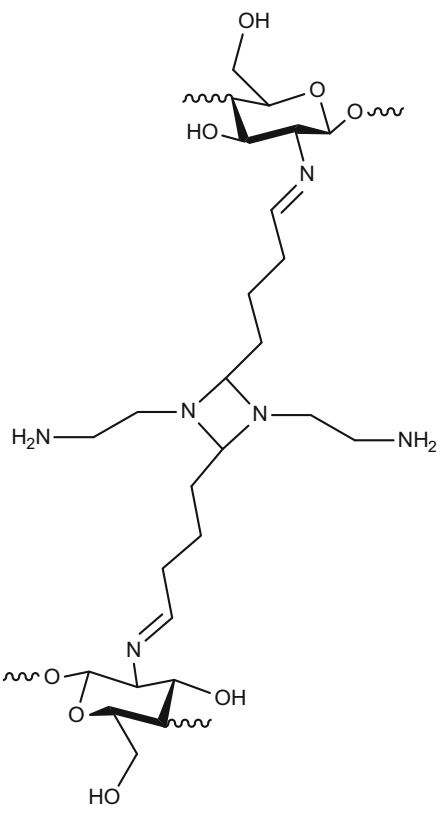


containing all constituent elements; volatilization is almost complete (with the exception of the CS-2 specimens); both endothermic and exothermic processes occur.

(c) In the case of nanocomposites containing magnetite (in both atmospheres) - the inorganic compound is undecomposed part of sample; there were no thermal effects which could be attributed to changes in the $\mathrm{Fe}_{3} \mathrm{O}_{4}$ in air, in contrast to an inert atmosphere. Magnetite exhibited catalytic action on thermal degradation of chitosan derivatives.

\section{Conclusions}

The several competitive reactions occur during heating of studied chitosan derivatives containing functional side chains. Besides processes typical for chitosan, also the reactions of amines and imines present in the substituents have to be taken into account. The high content of carbonaceous residue at $800{ }^{\circ} \mathrm{C}$ found in nitrogen arises from the carbonization process and formation of permanent cross-linked or polycyclic aromatic structures.

It was found that the lateral substituents, differing in the number and type of functional groups, determine the changes in the thermal degradation of the polymer. The substituent incorporated into macrochain decreases the thermal resistance of polysaccharide comparing to the pure chitosan, due to the relatively weak $\mathrm{C}-\mathrm{N}$ bonds.

The thermogravimetric analysis clearly indicates that the course of thermal degradation of chitosan containing two long substituents (CS-2) and final degradation products is different from the other studied systems. This specimen is characterized by the high content of strongly hydrogenbonded water, which is impossible to remove in drying at mild conditions. The adsorbed water is responsible for hydrolysis at elevated temperatures and has the effect of lowering of the overall thermal stability in this case.

Nanocomposites based on chitosan derivatives exhibited further decrease in thermal resistance, which indicates the catalytic action of magnetite. However, the thermal stability determined is still high enough for potential predicted applications. The $\mathrm{Fe}_{3} \mathrm{O}_{4}$-CS-3 nanocomposite exhibits the highest stability among the materials investigated in the present study.

The content of magnetite in the anhydrous nanocomposites, determined on the basis of the thermogravimetric measurements, is 11,10 and $13 \%$ in $\mathrm{Fe}_{3} \mathrm{O}_{4}-\mathrm{CS}-1, \mathrm{Fe}_{3} \mathrm{O}_{4}-$ CS-2 and $\mathrm{Fe}_{3} \mathrm{O}_{4}-\mathrm{CS}-3$, respectively.

Chitosan modified by the amino and imine groups is biologically active and can play important role in biotechnology and pharmacy; for example, it can be unique material for immobilization of enzymes and drugs. The presence of magnetite nanoparticles facilitates the separation of valuable and often very expensive bioactive compounds which allows for their multiple use.

Satisfactory thermal stability of the polymer nanoparticle matrices is important from the point of view of their application in catalysis, treatment of hyperthermia or other applications where materials designed to biomedical applications are subjected to heating.

Acknowledgements The project was supported by research Grant: National Science Centre 2014/13/B/ST8/04342.

Open Access This article is distributed under the terms of the Creative Commons Attribution 4.0 International License (http://crea tivecommons.org/licenses/by/4.0/), which permits unrestricted use, distribution, and reproduction in any medium, provided you give appropriate credit to the original author(s) and the source, provide a link to the Creative Commons license, and indicate if changes were made.

\section{References}

1. Mourya VK, Inamdar NN. Chitosan-modifications and applications: opportunities galore. React Funct Polym. 2008;68:1013-51.

2. Sashiwa H, Kawasaki N, Nakayama A, Muraki E, Yamamoto N, Aiba S. Chemical modification of chitosan. $14:^{1}$ Synthesis of water-soluble chitosan derivatives by simple acetylation. Biomacromolecules. 2002;3:1126-8.

3. Jeon C, Höll WH. Chemical modification of chitosan and equilibrium study for mercury ion removal. Water Res. 2003;37:4770-80.

4. Amjii MM. Surface modification of chitosan membranes by complexation-interpenetration of anionic polysaccharides for improved blood compatibility in hemodialysis. J Biomater Sci Polymer Edit. 1996;8:281-98.

5. Sashiwa H, Yamamori N, Ichinose Y, Sunamoto J, Aiba S. Michael reaction of chitosan with acrylic acid in water. Macromol Biosci. 2013;3:231-3.

6. Tangpauthadol V, Pongchaisirikul N, Hoven VP. Surface modification of chitosan films. Effects of hydrophobicity on protein adsorption. Carbohydr Res. 2003;38:937-42.

7. Amaral IF, Granja PL, Barbosa MA. Chemical modification of chitosan by phosphorylation: an XPS, FT-IR and SEM study. J Biomater Sci Polym Edit. 2005;16:1575-93.

8. Correia DM, Gámiz-González MA, Botelho G, Vidaurre A, Gomez Ribelles JL, Lanceros-Mendez S, Sencadas V. Effect of neutralization and cross-linking on the thermal degradation of chitosan electrospun membranes. J Therm Anal Calorim. 2014; 117:123-30.

9. Pereira FS, da Silva Agostini DL, Job AE, Gonzalez ERP. Thermal studies of chitin-chitosan derivatives. J Therm Anal Calorim. 2013;114:321-7.

10. Pawlak A, Mucha M. Thermal analysis of chitosan and its blends. Termochim Acta. 2003;396:153-66.

11. Maciel JS, Silva DA, Haroldo CB, de Paula RCM. Chitosan/carboxymethyl cashew gum polyelectrolyte complex: synthesis and thermal stability. Eur Polym J. 2005;41:2726-33.

12. Mucha M, Pawlak A. Thermal analysis of chitosan and its blends. Thermochim Acta. 2005;427:69-76. 
13. Ziegler-Borowska M, Siódmiak T, Chełminiak D, Cyganiuk A, Marszałł MP. Magnetic nanoparticles with surfaces modified with chitosan-poly[ $N$-benzyl-2-(methacryloxy)- $N, N$-dimethylethanaminium bromide] for lipase immobilization. Appl Surf Sci. 2014;288:641-8.

14. Ziegler-Borowska M, Chełminiak D, Siódmiak T, Sikora A, Marszałł MP, Kaczmarek H. Synthesis of new chitosan coated magnetic nanoparticles with surface modified with long-distanced amino groups as a support for bioligands binding. Mater Lett. 2014;132:63-5.

15. Siódmiak T, Ziegler-Borowska M, Marszałł MP. Lipase-immobilized magnetic chitosan nanoparticles for kinetic resolution of (R, S)-ibuprofen. J Mol Catal B. 2013;94:7-14.

16. Chełminiak D, Ziegler-Borowska M, Kaczmarek H. Polymer coated magnetite nanoparticles for biomedical applications. Part I. Preparation of nanoparticles $\mathrm{Fe}_{3} \mathrm{O}_{4}$ coated by polysaccharides. Polimery. 2015;60:12-7.

17. Ziegler-Borowska M, Chełminiak D, Kaczmarek H. Thermal stability of magnetic nanoparticles coated by blends of modified chitosan and poly(quaternary ammonium) salt. J Therm Anal Calorim. 2015;119:499-506.

18. Samejima K, Dairman W, Udenfriend S. Condensation of ninhydrin with aldehydes and primary amines to yield highly fluorescent ternary products. I. Studies on the mechanism of the reaction and some characteristics of the condensation product. Anal Biochem. 1971;42:222-36.

19. Heux L, Brugnerotto J, Desbieres J, Versali MF, Rinaudo M. Solid state NMR for determination of degree of acetylation of chitin and chitosan. Biomacromolecules. 2000;1:746-51.

20. Zeng L, Qin C, Wang L, Li W. Volatile compounds formed from the pyrolysis of chitosan. Carbohydr Polym. 2011;83:1553-7.

21. Fjaroh F, Setyawan H, Nur A, Lenggoro IW. Thermal stability of silica-coated magnetite nanoparticles prepared by an electrochemical method. Adv Powder Technol. 2013;24:507-11.

22. Carp O, Patron L, Culita DC, Budrugeac P, Feder M, Diamandescu L. Thermal analysis of two types dextran-coated magnetite. J Therm Anal Calorim. 2010;101:181-7.
23. Forsmo SPE. Oxidation of magnetite concentrate powders during storage and drying. Int J Miner Process. 2005;75:135-44.

24. Liang X, Zhong Y, Tan W, Zhy J, Yuan P, He H, Jiang Z. The influence of substituting metals ( $\mathrm{Ti}, \mathrm{V}, \mathrm{Cr}, \mathrm{Mn}, \mathrm{Co}$ and $\mathrm{Ni}$ ) on the thermal stability of magnetite. J Therm Anal Calorim. 2013;111:1317-24.

25. Monteiro DS, da GuardaSouza MO. Thermal decomposition of precursors and iron oxide properties. J Therm Anal Calorim. 2015;. doi:10.1007/s10973-015-4840-5.

26. Choudhury LH, Parvin T. Recent advances in the chemistry of imine-based multicomponent reactions (MCRs). Tetrahedron. 2011;67:8213-28.

27. Ferraris D. Catalytic, asymmetric alkylation of imines. Tetrahedron. 2007;63:9581-97.

28. Collin J, Jaber N, Lannou MI. Imino-Diels Alder and imino-aldol reactions catalyzed by samarium diiodide. Tetrahedron Lett. 2001;42:7405-7.

29. Zawadzki J, Kaczmarek H. Thermal treatment of chitosan in various conditions. Carbohydr Polym. 2010;80:395-401.

30. Martinez-Camacho AP, Cortez-Rocha MO, Ezquerra-Brauer JM, Graciano-Verdugo AZ, Rodriguez-Felix F, Castillo-Ortega MM, Yepis-Gomez MS, Plascencia-Jatomea M. Chitosan composite films: thermal, structural, mechanical and antifungal properties. Carbohydr Polym. 2010;82:305-15.

31. Hong PZ, Li SD, Ou CY, Li CP, Yang L, Zhang CH. Thermogravimetric analysis of chitosan. J Appl Polym Sci. 2007; 105:547-51.

32. R. Weast. CRC handbook of chemistry and physics. 69th ed. Boca Raton: CRC; 1989. http://www.organicchemistry.com/com bustion-heat-of-reaction-and-bond-energies/accessed. $15 \mathrm{Apr}$ 2015.

33. Zhang LM, Tan ZC, Wang SD, Wu DY. Combustion calorimetric and thermogravimetric studies of graphite and coals with a coalburning additive. Thermochim Acta. 1997;299:13-7. 